\title{
The Risk Factors For The Failure of Hook Wire Localization of Ground Glass Nodules Prior To Thoracoscopic Surgery
}

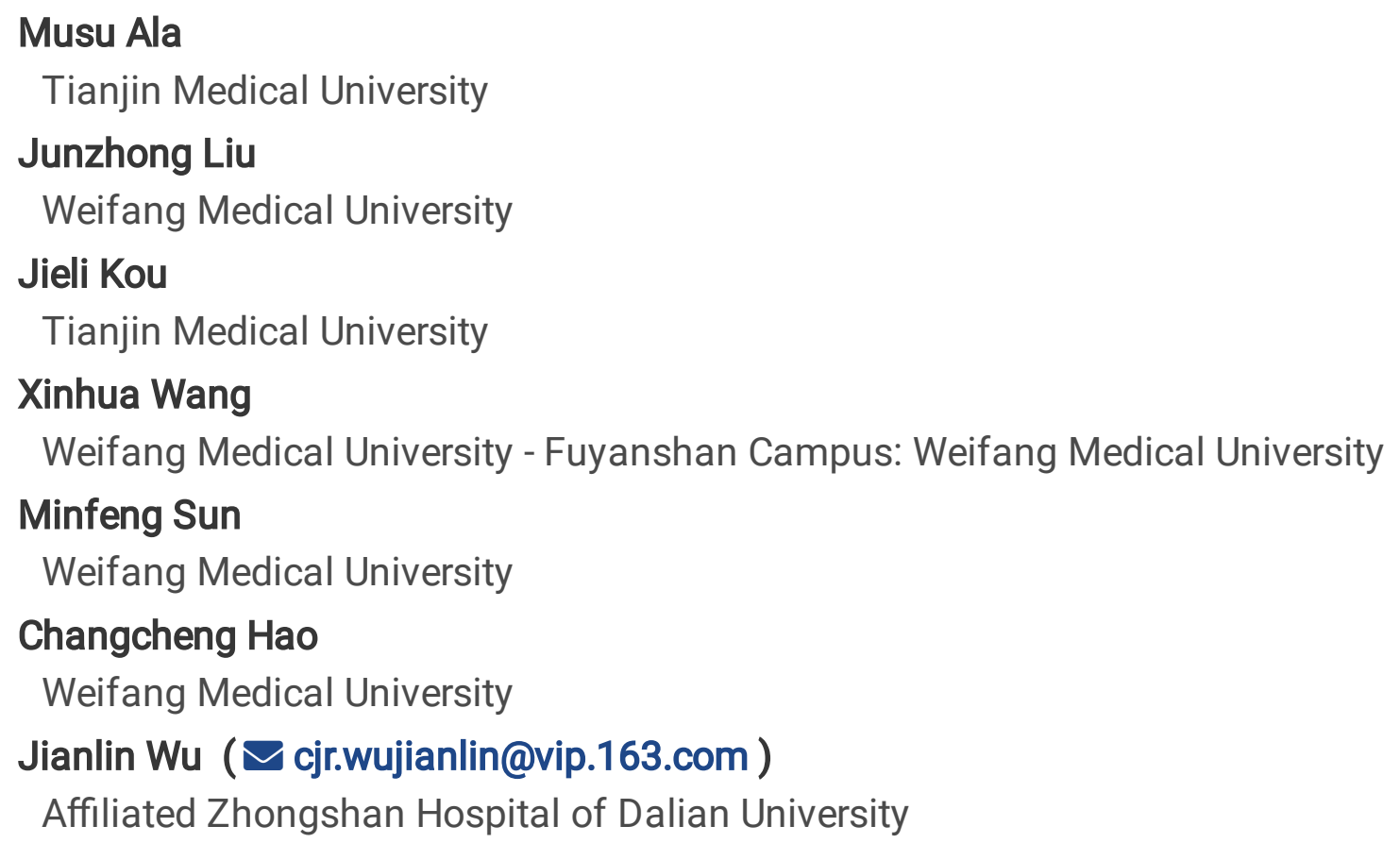

Musu Ala

Tianjin Medical University

Junzhong Liu

Weifang Medical University

Jieli Kou

Tianjin Medical University

Xinhua Wang

Weifang Medical University - Fuyanshan Campus: Weifang Medical University

Minfeng Sun

Weifang Medical University

Changcheng Hao

Weifang Medical University

Jianlin Wu ( $\nabla$ cjr.wujianlin@vip.163.com )

Affiliated Zhongshan Hospital of Dalian University

Research article

Keywords: Pneumothorax, localization, ground glass nodule, thoracoscopic surgery

Posted Date: September 20th, 2021

DOI: https://doi.org/10.21203/rs.3.rs-842171/v1

License: (c) (i) This work is licensed under a Creative Commons Attribution 4.0 International License. Read Full License 


\section{Abstract}

Objectives: To retrospectively analyse the potential influencing factors of CT-guided hook wire localization failure prior to thoracoscopic resection surgery of ground glass nodules (GGNs), and determine the main risk elements for localization failure.

Methods: In all, 372 patients were included in this study, with 21 patients showing localization failure. The related parameters of patients, GGNs, and localization were analysed through univariate and multiple logistic regression analysis to determine the risk factors of localization failure.

Results: Univariate logistic regression analysis indicated that trans-fissure (odds ratio [OR]: $4.896,95 \%$ confidence interval [CI]: 1.489-13.939); trans-emphysema (OR: 3.538, 95\% Cl: 1.343-8.827); localization time (OR: 0.956, 95\%Cl: 0.898-1.019); multi-nodule localization (OR: 2.597, 95\%Cl: 1.050-6.361); and pneumothorax (OR: $10.326,95 \% \mathrm{Cl}$ : $3.414-44.684$ ) were risk factors for localization failure, and the $\mathrm{p}$ values of these factors were $<0.05$. However, according to the results of multivariate analysis, pneumothorax (OR: 5.998, 95\% Cl: 1.680-28.342) was an exclusive risk factor for the failure of preoperative localization of GGNs.

Conclusion: CT-guided hook wire localization of GGNs prior to thoracoscopic surgery is often known to fail; however, the incidence is low. Pneumothorax is an independent risk factor for failure in the localization process.

\section{Background}

Early resection of pulmonary ground glass nodules (GGNs) with malignant signs can improve the survival rate of patients[[1]]. Video-assisted thoracoscopic surgery (VATS) is a recognized and effective option to resect malignant GGNs early, and has been widely applied given that it is less invasive than traditional open thoracotomy [[2-4]]. Because of the soft texture of GGNs, it is difficult for thoracic surgeons to touch the nodules with their fingers[5]. Therefore, it is necessary to locate the hook wire marker under the guidance of computed tomography (CT) to achieve precise localization and resection of GGNs through VATS $[5,6]$.

Hook wire is one of the most commonly used preoperative markers that has a high success rate[4, 7, 8]. However, there are still risks of localization failure, such as dislodgement or displacement[9, 10], which likely affect the precise removal of pulmonary nodules in thoracoscopic surgery. Despite this, there are few reports on the failure of preoperative localization of pulmonary nodules[11, 12].

In this paper, we retrospectively analysed the potential risk factors of failure to localize the GGNs under CT guidance prior to VATS and determined predictors for localization failure.

\section{Methods And Materials}


All patients signed the informed consent before CT-guided localization of GGNs. Because all the data are anonymous, our institutional review board allowed this retrospective study and waived the need for informed consent to collect and use the patients' data.

\section{Patients}

From January 2014 to December 2019, a total of 372 patients (single nodules, $n=351$; multiple nodules, $n=21$ ) who underwent preoperative hook wire localization before VATS surgery were enrolled in this study. The inclusion criteria were as follows: (1) simultaneous localization of single or multiple GGNs in unilateral lung; (2) hook wire localization; (3) subpleural measurement of GGNs > $5 \mathrm{~mm}$ and/or $<40 \mathrm{~mm}$; and (4) both successful and failed localization cases. The exclusion criteria were as follows: (1) depth of GGNs > $40 \mathrm{~mm}$ or < $5 \mathrm{~mm}$; (2) bilateral pulmonary nodules localized simultaneously; (3) large blood vessels or bronchi around or across the nodules; and (4) other localization markers. The enrolled patients were divided into the success and failure groups. The flowchart of the study population is exhibited presented in Fig. 1.

Hook wire localization

An attending radiologist with five years' experience completed the process of localization of GGNs in collaboration with the thoracic surgeon. The localization set of the hook wire was $20 \mathrm{G} \times 120 \mathrm{~mm}$ (Pajunk, Germany). The 64-slice spiral CT used for preoperative localization was manufactured by GE Healthcare (Connecticut State, USA).

After ensuring the appropriate body position and localization path based on the previous CT images, the patient underwent preoperative marker localization process of GGNs under local anaesthesia along the shortest localization pathway as far as possible. Once the tip of the guide needle reached around the target nodule, the hook wire was released and the guide needle withdrawn. If the needle tip did not reach the nodule position, the guide needle was re-adjusted to the ideal position. The ideal position is to locate the hook wire around or cross the target nodule within $1 \mathrm{~cm}$. After withdrawal of the guide needle, the CT is repeated to confirm whether the localization is successful (Fig. 2A). If the localization operation is successful, the patient will be sent to the operating room accompanied by a nurse within $1 \mathrm{~h}$. However, if the procedure fails by way of dislodgement or displacement, the localization operation will be retried with the patient's permission, regardless of whether the localization is successful. The results of localization need to be relayed to the thoracic surgeon, so that the surgical method can be determined according to the situation.

\section{VATS}

The patients underwent dual-port thoracoscopic resection in a suitable position under general anaesthesia. The VATS operation is performed by visualizing the end of the hook wire on the pleural surface under the guidance of nodule localization (Fig. 2B). The choice between lobectomy and wedge resection is made by the thoracic surgeon. Next, the resected specimens are sent to the pathology 
department for a quick frozen-section examination to confirm whether the resected lesions are benign or malignant (Fig. 2C). Hilar and mediastinal lymph nodes are usually dissected, and the nature of the nodes are confirmed pathologically.

Data collection and variables definition

The baseline information of all patients was collected by an attending radiologist through the Picture Archiving and Communication Systems, and the imaging data was measured and recorded on the GE Advantage Workstation (v.4.6). The baseline variables include the patient's age, sex, and smoking status. The parameters of GGNs are as follows: diameter; depth of GGN (in millimetres from the target nodule to the nearest pleura [the depth is recorded according to the shallowest nodule when multiple GGNs are localized]); nodule position (upper and middle or lower lobe); and whether multiple GGNs are localized. The localization-related variables are puncture position (supine, prone, or lateral); trans-emphysema (in the localization pathway); trans-fissure (transfissural approach in the localization pathway); location time; pneumothorax; pulmonary haemorrhage (localization related); and localization result (success or failure).

The definition of placement failure is as follows: (1) The hook wire marker could not be successfully released during the localization process; (2) although the hook wire was successfully placed on the target nodule for the first time or once again, the dislodgement or decoupling occurred during CT confirmation after the localization; (3) preoperative localization of the hook wire was accurate and successful under the CT guidance, but the marker was found to fall off during thoracoscopic surgery.

Statistical analysis

All 372 patients were divided into two groups according to whether the localization was successful, and the risk factors of localization failure were assessed. The variable factors that were established to be different in the univariate regression analysis were incorporated into the multiple logistic regression analysis to identify the determinants of the localization failure. The diagnostic ratio (OR) and $95 \%$ confidence interval $(\mathrm{Cl})$ of each independent factor was calculated. Further, $\mathrm{p}<0.05$ was considered to indicate statistical significance. SPSS software (v.25, IBM Corporation, Armonk, NY, USA) was utilized for statistical analyses.

\section{Results}

The baseline characteristics of the participants and parameters related to localization are shown in Table 1. Among the 372 patients who were localized before VATS, 351 patients were successfully localized, and the other 21 cases showed failure, of which eight cases were attributed to dislodgement. When repeating the CT scan to confirm the position after localization, it was found that hook wires protruded from the lung parenchyma and were accompanied by pneumothorax in five out of eight cases. The other three cases showed dislodgement and pneumothorax during thoracoscopic surgery after being sent to the operating room after successful preoperative localization. The reason for the remaining 13 cases of failure was that pneumothorax occurred during the localization process and could not be accurately 
localized, although relocation was attempted. For the cases that could not be located, segmental resections were done by the hematoma on the pleural surface in three cases, and the remaining cases underwent lobectomy under VATS. 
Table 1

the baseline characteristics of the participants and parameters related to localization

\begin{tabular}{|c|c|c|c|}
\hline Variable & $\begin{array}{l}\text { Success } \\
(n=351)\end{array}$ & $\begin{array}{l}\text { Failure } \\
(n=21)\end{array}$ & P-value \\
\hline Age & $58.95 \pm 9.96$ & $59.62 \pm 10.33$ & 0.764 \\
\hline Sex & & & 0.825 \\
\hline Male & $179(51.0 \%)$ & $10(47.6 \%)$ & \\
\hline Female & $172(49.0 \%)$ & $11(52.4 \%)$ & \\
\hline Smoke & & & 0.532 \\
\hline No & $256(72.9 \%)$ & $14(66.7 \%)$ & \\
\hline Yes & $95(27.1 \%)$ & $7(33.3 \%)$ & \\
\hline Localization of multiple nodules & & & 0.030 \\
\hline No & $260(74.1 \%)$ & $11(52.4 \%)$ & \\
\hline Yes & $91(25.9 \%)$ & $10(47.6 \%)$ & \\
\hline Localization time (min) & $22.20 \pm 10.52$ & $19.14 \pm 7.16$ & 0.190 \\
\hline Depth of nodule (mm) & $21.34 \pm 9.17$ & $15.52 \pm 6.85$ & 0.005 \\
\hline Diameter (mm) & $8.99 \pm 3.80$ & $9.29 \pm 3.47$ & 0.732 \\
\hline Trans-fissure & & & 0.002 \\
\hline No & $329(94.0 \%)$ & $16(72.2 \%)$ & \\
\hline Yes & $21(6.0 \%)$ & $5(23.8 \%)$ & \\
\hline Trans-emphysema & & & 0.005 \\
\hline No & $299(85.2 \%)$ & $13(14.8 \%)$ & \\
\hline Yes & $52(69.9 \%)$ & $8(38.1 \%)$ & \\
\hline Puncture position & & & 0.450 \\
\hline Supine & 112 (31.9\%) & $9(42.9 \%)$ & \\
\hline Prone & $153(43.6 \%)$ & $9(42.9 \%)$ & \\
\hline Lateral & $86(24.5)$ & $3(14.3 \%)$ & \\
\hline Location & & & 0.737 \\
\hline Upper and middle & $197(56.1 \%)$ & $11(52.4 \%)$ & \\
\hline
\end{tabular}




\begin{tabular}{|llll|}
\hline Variable & $\begin{array}{l}\text { Success } \\
(\mathbf{n = 3 5 1 )}\end{array}$ & $\begin{array}{l}\text { Failure } \\
(\mathbf{n}=\mathbf{2 1})\end{array}$ & P-value \\
\hline lower & $154(43.9 \%)$ & $10(47.6 \%)$ & \\
\hline Pneumothorax & & & $<0.001$ \\
\hline No & $222(63.2 \%)$ & $3(14.3 \%)$ & \\
\hline Yes & $129(36.8 \%)$ & $18(85.7 \%)$ & \\
\hline Pulmonary hemorrhage & & & 0.075 \\
\hline No & $301(85.8 \%)$ & $15(71.4 \%)$ & \\
\hline Yes & $50(14.2 \%)$ & $6(28.6 \%)$ & \\
\hline
\end{tabular}

Pneumothorax occurred in $39.52 \%$ (147/372) of all localization patients, and the rate in the failure group was $87.5 \%$ (18/21) during the CT-guided localization process. Univariate logistic regression analysis indicated that trans-fissure (OR 4.896, 95\% Cl: 1.489-13.939); trans-emphysema (OR: $3.538,95 \% \mathrm{Cl}$ : 1.343-8.827); localization time (OR: $0.956,95 \% \mathrm{Cl}$ : 0.898-1.019): multi-nodule localization (OR: 2.597 , 95\%Cl: 1.050-6.361); and pneumothorax (OR: 10.326, 95\%Cl: 3.414-44.684) were risk factors for localization failure, and the $p$-values of these factors were $<0.05$. However, according to the results of multivariate analysis, pneumothorax was an exclusive risk factor for the failure of preoperative localization of GGNs. The results of univariate and multivariate regression analysis are presented in Table 2. 
Table 2

univariate and multivariable analysis of baseline and CT features

Univariable analysis

Multivariable analysis

\begin{tabular}{|c|c|c|c|c|c|c|}
\hline & OR & $95 \% \mathrm{Cl}$ & $\begin{array}{l}P \\
\text { value }\end{array}$ & OR & $95 \% \mathrm{Cl}$ & $\begin{array}{l}P \\
\text { value }\end{array}$ \\
\hline Age & 1.007 & $0.963-1.053$ & 0.763 & & & \\
\hline \multicolumn{7}{|l|}{ Sex } \\
\hline Male & 1.0 & & & & & \\
\hline Female & 1.145 & $0.471-2.814$ & 0.764 & & & \\
\hline \multicolumn{7}{|l|}{ Smoke } \\
\hline No & 1.0 & & & & & \\
\hline Yes & 1.347 & $0.528-3.440$ & 0.533 & & & \\
\hline \multicolumn{7}{|l|}{$\begin{array}{l}\text { Localization of multiple } \\
\text { nodules }\end{array}$} \\
\hline No & 1.0 & & & & & \\
\hline Yes & 2.597 & $1.050-6.361$ & 0.035 & 1.346 & $\begin{array}{l}0.494- \\
3.595\end{array}$ & 0.552 \\
\hline Location time (min) & 0.956 & $0.898-1.019$ & 0.166 & & & \\
\hline Depth of nodule (mm) & 0.910 & $0.850-0.973$ & 0.006 & 0.942 & $\begin{array}{l}0.875- \\
1.014\end{array}$ & 0.113 \\
\hline \multicolumn{7}{|l|}{ Trans-fissure } \\
\hline No & 1.0 & & & & & \\
\hline Yes & 4.896 & $\begin{array}{l}1.489- \\
13.939\end{array}$ & 0.005 & 2.168 & $\begin{array}{l}0.613- \\
6.773\end{array}$ & 0.199 \\
\hline \multicolumn{7}{|l|}{ Trans-emphysema } \\
\hline No & 1.0 & & & & & \\
\hline Yes & 3.538 & $1.343-8.827$ & 0.008 & 1.003 & $\begin{array}{l}0.343- \\
2.806\end{array}$ & 0.995 \\
\hline Diameter (mm) & 1.020 & $0.912-1.141$ & 0.731 & & & \\
\hline \multicolumn{7}{|l|}{ Insertion position } \\
\hline Supine & 1.0 & & & & & \\
\hline Prone & 2.304 & $0.605-8.767$ & 0.221 & & & \\
\hline
\end{tabular}




$\begin{array}{llll}\text { lateral } & 1.686 & 0.445-6.396 & 0.442\end{array}$

Location

Upper and middle

1.0

Lower

0.860

$0.356-2.077$

0.737

Pneumothorax

No

1.0

Yes

10.326

$3.414-$

44.684

$<$

0.001

5.998

$1.680-$

28.342

0.010

Pulmonary hemorrhage

No

1.0

Yes

1.552

$0.909-2.497$

0.083

\section{Discussion}

A variety of preoperative markers for GGNs prior to VATS have been reported, including hook wire[4, 13], coil[14, 15], methyl blue[16], 99mTc[17], hydrogel plugs[18], barium[8, 9], and fiducial marker[8, 19]. Because of its high feasibility and usability, hook wire is the most widely used marker in preoperative localization. Although the preoperative hook wire localization has a high success rate, the reported probability of hook wire localization failure is $7.5 \%$ owing to migration or dislodgement $[4,9]$. At present, there are few articles on the analysis of localization failure[11]. Therefore, the purpose of this article was to clarify the factors of localization failure to provide clinical decision-making in advance.

In this study, 21 out of 372 patients showed localization failure with an incidence rate of $5.65 \%$, which is similar to that reported in the literature[9]. Among the failed cases, 13 developed pneumothorax during the positioning process. As the pneumothorax progresses, the tip of the guide wire protrudes out of the lung parenchyma, and the hook wire cannot be released to the target nodule. Among the 21 failed cases, 13 showed pneumothorax before the guide wire was released successfully during the localization process. As the pneumothorax progresses, the guide wire tip protrudes out of the pleural cavity and the hook wire cannot be released to the target nodule. Even if the positioning is reinitiated, it is difficult to fix the guide needle to the ideal position within the calculated distance given the pneumothorax, which causes the swing of the pleural cavity. Usually, when pneumothorax occurs, the depth of localization is longer than the predetermined distance given the retraction of the lung tissue. However, it is difficult for the doctor to insert the guide needle excessively beyond the predetermined distance during operation to avoid damage to large blood vessels and cause severe haemoptysis. The remaining eight failed cases of 
dislodgement were found with pneumothorax during CT reconfirmation (five cases) and VATS (three cases) after the initial successful localization.

In our study, the incidence of pneumothorax formation is $39.52 \%$, which is within the range of $12.8-$ $68.1 \%$ reported in the literature[11]. In this study, $85.7 \%$ of localization failure cases were related to pneumothorax $(\mathrm{P}<0.001$ compared with the control group). The results of multiple regression analysis also confirmed that pneumothorax was the independent factor related to localization failure. Literature reports show that trans-fissure is an important influencing factor of localization failure[10, 11]. In our results, trans-fissure showed a significant difference in the univariate logistic analysis. It is an important influencing factor rather than an independent factor, which is different from the literature reports. Because the trans-fissure approach has to penetrate three layers of pleura, the incidence of pneumothorax with the trans-fissure approach is higher than that with the traditional approach. We speculate that the trans-fissure approach is due to the occurrence of pneumothorax and leads to localization failure; hence, the trans-fissure is a risk factor for pneumothorax, not a direct risk factor for localization failure.

According to the results of univariate logistic regression, other factors such as depth of the nodule, transemphysema, and localization of multiple nodules are also related to the failure of localization. The shallower the location depth, the greater the risk of dislodgment after the occurrence of pneumothorax, because the tip of the hook wire is easily pulled out of the pulmonary parenchyma under the swing of the chest cavity. Previous studies have shown that the shallower depth threshold for hook wire placement is $1.8 \mathrm{~cm}[10]$, so as to possibly avoid pneumothorax. In our research, the localization depth of the failure group was shallower than that of the success group $(15.52 \pm 6.85 v s .21 .34 \pm 9.17, p<0.001)$. Although published studies have reported that it is safe and feasible to locate multiple nodules at the same time[20], our study found that the rate of failure was higher than that of the single nodule group $(47.6 \%$ vs. $25.9 \%, p=0.030$ ). Simultaneous localization of multiple nodules is related to localization failure, which may increase the risk of localization failure. Emphysema, especially trans-emphysema or pulmonary bullae in the puncture path, is the influencing factor of localization failure according to the results of our univariate logistic regression analysis. Therefore, the pre-localization plan should attempt to avoid emphysema in the lung parenchyma.

Other reported factors of localization are the location of the nodule in the lower lobe[21], surgical history[21], vital capacity[11, 22], physician experience[11], and location time[10, 23]. These factors are mainly related to the formation of pneumothorax and whether they are related to localization failure still requires further investigation. The results of multiple regression analysis in this study indicate that only pneumothorax is an independent risk factor. This could be because the tail of the hook wire is fixed on the chest wall and the hook tip that is placed on the target nodule can move relative to each other with the formation and progression of pneumothorax. The formation of pneumothorax can drag the hook wire that has been fixed to the target nodule. The hook wire tip may be displaced or pulled off into the chest cavity, resulting in failure of localization. 
The limitation of this article is that the sample size of the failure group is quite small, which maybe affect the results of statistical analysis, and there is no comparison and inclusion of other commonly used metal markers to analyse the causes of failure, such as Coil.

In conclusion, although the preoperative localization success rate of GGNs under CT guidance is high, localization failure still exists. Pneumothorax is an independent risk factor for failure in the localization process.

\section{Abbreviations}

GGN:Ground Glass Nodule; Cl: Confidence Interval; OR: Odds Ratio; VATS: Video Assisted Thoracoscopic Surgery; CT: Computed Tomography

\section{Declarations}

\section{Availability of data and materials}

The datasets used and/or analyzed during the current study are available from the corresponding author on reasonable request.

\section{Acknowledgments}

We would like to give my grateful to Dr. Junzhong Liu for his endless help and support in his impactful research suggestions and statistical analysis consultation for this project.

And we thank Charlesworth Author Services for the language editor of the manuscript.

\section{Funding}

This research was supported by the Weifang Science and Technology Development Plan (2020YX073).

\section{Ethics declarations}

\section{Ethics approval and consent to participate}

This study was conducted in accordance with the declaration of Helsinki.

This study was approved by the Ethics Committee of the Second People's Hospital of Weifang. Because this was a retrospective study, informed consent was waived.

\section{Consent for publication}

We have obtained consent to publish from the participants (or legal parent or guardian) to report their individual patient data. 


\section{Competing interests}

The authors declare that they have no competing interests.

\section{References}

1. Zhang Y, Fu F, Chen $\mathrm{H}$. Management of ground-glass opacities in the lung cancer spectrum. Ann Thoracic Surg. 2020;110(6):1796-804.

2. Burdine J, Joyce LD, Plunkett MB, et al. Feasibility and value of video-assisted thoracoscopic surgery wedge excision of small pulmonary nodules in patients with malignancy. Chest. 2002;122(4):146770 .

3. Mun M, Kohno T. Efficacy of thoracoscopic resection for multifocal bronchioloalveolar carcinoma showing pure ground-glass opacities of $20 \mathrm{~mm}$ or less in diameter. J Thoracic Cardiovasc Surg. 2007;134(4):877-82.

4. Liu J, Wang $X$, Wang Y, et al. Comparison of CT-guided localization using hook wire or coil before thoracoscopic surgery for ground glass nodules. Br J Radiol. 2020;93(1111):20190956.

5. Nakashima S, Watanabe A, Obama T, et al. Need for preoperative computed tomography-guided localization in video-assisted thoracoscopic surgery pulmonary resections of metastatic pulmonary nodules. Ann Thorac Surg. 2010;89(1):212-8.

6. Huang $\mathrm{W}, \mathrm{Ye} \mathrm{H}, \mathrm{Wu} \mathrm{Y}$, et al. Hook wire localization of pulmonary pure ground-glass opacities for video-assisted thoracoscopic surgery. Thoracic Cardiovasc Surg. 2014;62(2):174-8.

7. Huang HZ, Wang GZ, Xu LC, et al. CT-guided Hookwire localization before video-assisted thoracoscopic surgery for solitary ground-glass opacity. Oncotarget. 2017;8(64):108118-29.

8. McDermott S, Fintelmann FJ, Bierhals AJ, et al. Image-guided preoperative localization of pulmonary nodules for video-assisted and robotically assisted surgery. Radiographics. 2019;39(5):1264-79.

9. Park $\mathrm{CH}, \mathrm{Han} \mathrm{K}, \mathrm{Hur} \mathrm{J}$, et al. Comparative effectiveness and safety of preoperative lung localization for pulmonary nodules: a systematic review and meta-analysis. Chest. 2017;151(2):316-28.

10. Liu J, Liang C, Wang X, et al. A computed tomography-based nomogram to predict pneumothorax caused by preoperative localization of ground glass nodules using hook wire. $\mathrm{Br} \mathrm{J}$ Radiol. 2021;94(1117):20200633.

11. Iguchi T, Hiraki T, Matsui $Y_{\text {, }}$ et al. Preoperative short hookwire placement for small pulmonary lesions: evaluation of technical success and risk factors for initial placement failure. Eur Radiol. 2018;28(5):2194-202.

12. Iguchi T, Hiraki T, Gobara H, et al. CT fluoroscopy-guided preoperative short hook wire placement for small pulmonary lesions: evaluation of safety and identification of risk factors for pneumothorax. Eur Radiol. 2016;26(1):114-21.

13. Li C, Liu B, Jia H, et al. Computed tomography-guided hook wire localization facilitates videoassisted thoracoscopic surgery of pulmonary ground-glass nodules. Thorac Cancer. 
2018;9(9):1145-50.

14. Donahoe LL, Nguyen ET, Chung TB, et al. CT-guided microcoil VATS resection of lung nodules: a single-centre experience and review of the literature. J Thorac Dis. 2016;8(8):1986-94.

15. McGuire AL, Vieira A, Grant K, et al. Computed tomography-guided platinum microcoil lung surgery: a cross-sectional study. J Thorac Cardiovasc Surg. 2019;158(2):594-600.

16. Lin CW, Ko HJ, Yang SM, et al. Computed tomography-guided dual localization with microcoil and patent blue vital dye for deep-seated pulmonary nodules in thoracoscopic surgery. J Formos Med Assoc. 2019;118(6):979-85.

17. Nardini M, Bilancia R, Paul I, et al. (99m)Technetium and methylene blue guided pulmonary nodules resections: preliminary British experience. J Thorac Dis. 2018;10(2):1015-21.

18. Imperatori A, Fontana F, Dominioni L, et al. Video-assisted thoracoscopic resection of lung nodules localized with a hydrogel plug. Interact Cardiovasc Thorac Surg. 2019;29(1):137-43.

19. Sharma A, McDermott S, Mathisen DJ, et al. Preoperative localization of lung nodules with fiducial markers: feasibility and technical considerations. Ann Thoracic Surg. 2017;103(4):1114-20.

20. Yan $Z, X u X Q, P a n X L$, et al. retrospective evaluation of safety, efficacy and risk factors for pneumothorax in simultaneous localizations of multiple pulmonary nodules using hook wire system. Cardiovasc Intervent Radiol. 2017;40(9):1408-14.

21. Hiraki T, Mimura $H$, Gobara $H$, et al. Incidence of and risk factors for pneumothorax and chest tube placement after CT fluoroscopy-guided percutaneous lung biopsy: retrospective analysis of the procedures conducted over a 9-year period. Am J Roentgenol. 2010;194(3):809-14.

22. Saji $H$, Nakamura $H$, Tsuchida $T$, et al. The incidence and the risk of pneumothorax and chest tube placement after percutaneous CT-guided lung biopsy: the angle of the needle trajectory is a novel predictor. Chest. 2002;121(5):1521-6.

23. Khan MF, Straub R, Moghaddam SR, et al. Variables affecting the risk of pneumothorax and intrapulmonal hemorrhage in CT-guided transthoracic biopsy. Eur Radiol. 2008;18(7):1356-63.

\section{Figures}




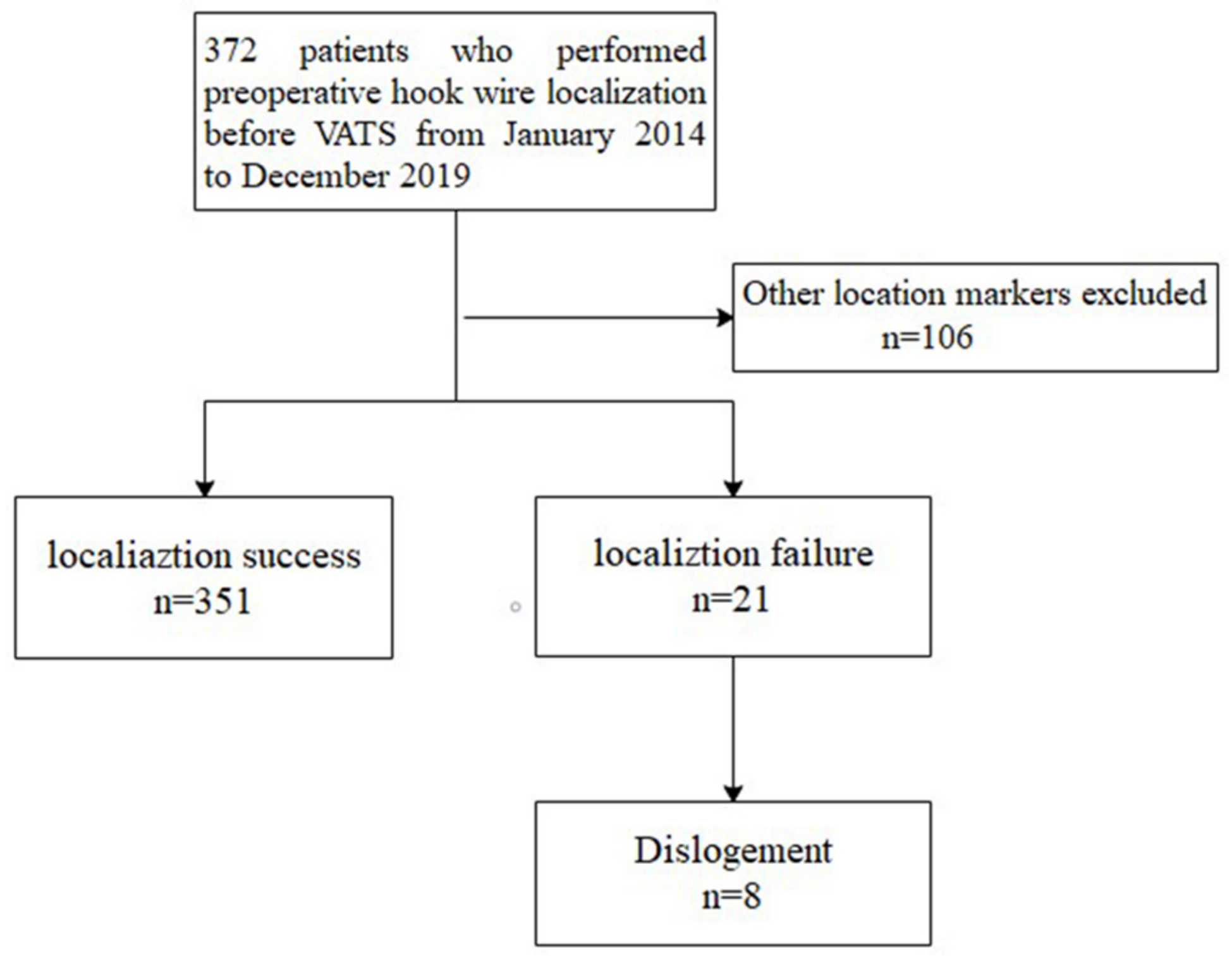

Figure 1

Flowchart of the study population.
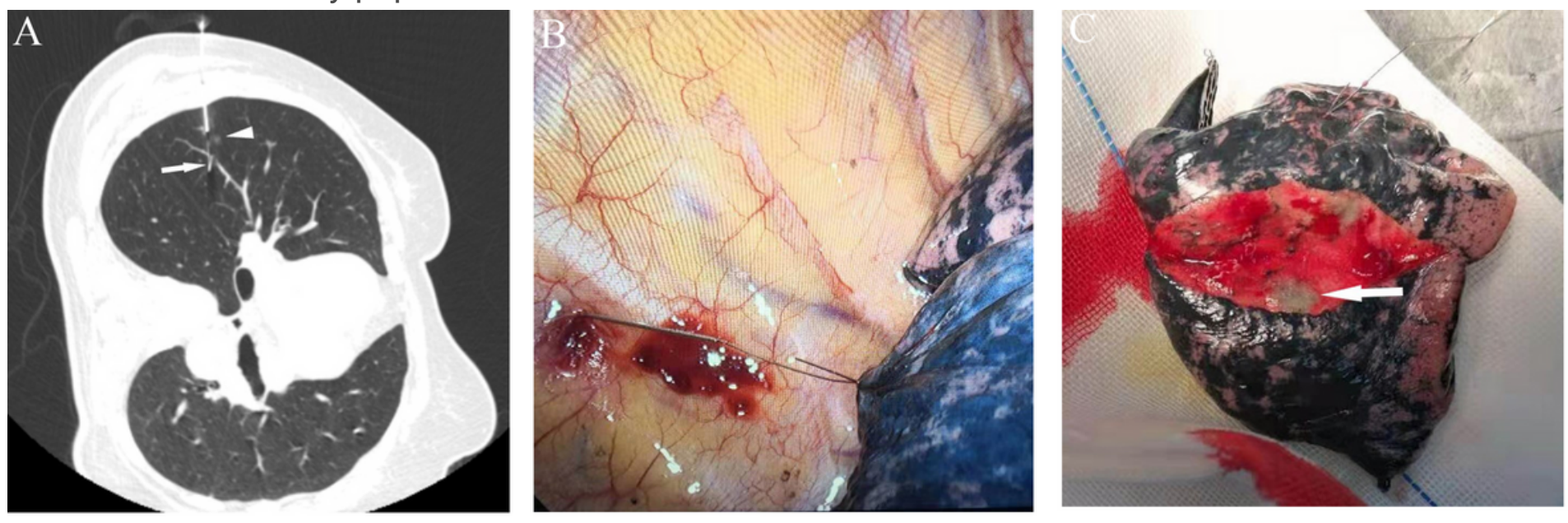


\section{Figure 2}

58-year-old woman who underwent hook wire localization prior to VATS. A) Hook wire (white arrow) was positioned next to the ground glass nodule (white triangle arrow) of the right upper lobe before thoracoscopic surgery. B) Hook wire was seen on the pleura surface during thoracoscopic surgery. C) The main pathology of the lesion after thoracoscopic resection (the white arrow shown the ground glass nodule). 